\title{
Swimming of Onboard-Powered Autonomous Robots in Viscous Fluid Filled Channels
}

\author{
Aydek Gokce Erman ${ }^{1}$, Serhat Yesilyurt ${ }^{2}$ \\ Mechatronics Program, Sabanci University, Istanbul, Turkey \\ ${ }^{1}$ gokceelsabanciuniv.edu \\ ${ }^{2}$ syesilyurtesabanciuniv.edu
}

\begin{abstract}
Microrobots can make a great impact in medical applications such as minimally-invasive surgery, screening and diagnosis of diseases, targeted therapy and drug delivery. Smallsized bio-inspired robots can mimic flagellar propulsion mechanisms of microorganisms for actuation in microfluidic environments, which are dominated by viscous forces. Microorganisms propel themselves by means of the motion of their flagella such as rotation of rigid helices or travelling planar waves on flexible tails similar to whipping motion. Here, we present characterization of swimming of onboard-powered autonomous robots inside cylindrical tubes. Robots consist of two links, head and tail, connected with a revolute joint. Rigid helical tails of the swimmer robots are made of steel wires with 12 different configurations of helical radius and pitch. From experiments forward linear velocity of robots and angular velocities of the links are measured, and compared with the mathematical model, which is based on the resistive force theory. Results indicate that the motion of the swimmer inside channels can be predicted by means of the resistive force theory reasonably well.
\end{abstract}

Keywords - autonomous, micro swimmer, helical wave propagation, resistive force theory, Stokes flow

\section{INTRODUCTION}

Size is one of the most important issues in microrobot technology since creating microrobots with smaller size will increase the ability to reach and operate inside the human body [1]. Moreover microrobots will improve minimally invasive surgery, screening and diagnosis of diseases, targeted therapy and drug delivery. On the other side, producing effective robots in small size is a difficult task due to generation and storage of power issue, lack of fabrication technique and lack of adaptation ability to alternating human body conditions. Thus, it is important to find the most efficient model for microswimming.

Swimming in micro scales requires different techniques than in macro scales due to the dominance of viscous forces in micro flows as opposed to the inertial ones in macro flows. Due to the resistive nature of the viscous forces, in principle, the relationship between the forces and velocities can be cast in that form: Gray and Hancock used the resistive force theory (RFT) to obtain the forward velocity of a micro organism based on the slender body theory (SBT) [2]. Later, Sir Lighthill improved the SBT with an elaborate analysis of the flow field enacted by the motion of the flagellum and long range hydrodynamic interactions [3]. In SBT, the resistive force coefficients are calculated with respect to geometry of the object and considering the hydrodynamic interactions of the
Stokeslet distribution. Keller and Rubinow proposed a full six degree of freedom rigid body equations of motion for the trajectory of microorganisms with simplifying assumptions in calculation of the rotation matrices and simple resistive force coefficients [4].

Previous experimental work with microrobots and robots swimming in viscous fluids vary with respect to size, propulsion mechanism, fabrication, actuation and assembly. Sendoh et al. produced a spiral type magnetic micro machine and generated backward and forward thrust by adjusting the direction of magnetic field [5]. Guo et al. designed a 3 DOF fish like robot with $45 \mathrm{~mm}$ in length, $10 \mathrm{~mm}$ in width and $4 \mathrm{~mm}$ in thickness, which was actuated by ICPF (Ionic Conducting Polymer Film) in water or aqueous medium [6]. Also, Kim et al. implemented a tadpole design with one polymer fin tail, which was driven by IPMC actuator in water [7]. Dreyfus et al. produced an artificial flagellum which was attached to a red blood cell and controlled by a time varying magnetic field [8]. An E.coli inspired propulsive mechanism was proposed by Behkam and Sitti, and actuated by two phase stepper motor, to calculate thrust force, which is measured by the deflection of the beam [9]. Authors also proposed that on board actuation can be achieved by using flagellar motors inside the intact cell of Serratia Marcescens bacteria [10]. An artificial bacterial flagella with helical tail was introduced by Zhang et al., which was controlled by a low strength rotating magnetic field [11]. Chattopadhyay and $\mathrm{Wu}$ showed that local resistive force theory could not describe well the dynamic of single cell swimming due to assumption of a stationary background by comparing swimming properties of different bacteria with including long range body interaction calculations [12].

For developing fully miniaturized wireless microrobots, power issue must be considered for producing a practical design [1]. For on board power, rechargeable thin film batteries can be used due to have their high energy densities, ability to fabricate into a variety of sizes and shapes on any type of substrate within wide temperature range operation limit [13]. By planar fabrication process arbitrarily shaped batteries with thickness less than $50 \mu \mathrm{m}$ can be produced [1].

Experimental characterization of swimming of microrobots inside cylindrical tubes with on-board power sources is presented here. Our experiments are motivated by the need of understanding of the motion of the swimming microrobots inside tubes and channels, which targets application of microswimming robots in medicine, such as the motion inside veins, arteries and other conduits in the body. Experiments are con- 
ducted in a silicon-oil filled glass tubes using 12 different configurations of rigid helical flagella attached to a glass capsule which has a diameter of $15.8 \mathrm{~mm}$ diameter, and length of $40 \mathrm{~mm}$. Results are compared with a simple mathematical model that incorporates resistive force coefficients from Sir Lighthill's analysis [3] to obtain the propulsion forces of tails as a function of helical pitch and radii.

\section{EXPERIMENTAL METHOD}

The onboard-powered swimming robot has two components (links): body and tail. The body of the robot is made from glass tube having $15.8 \mathrm{~mm}$ in diameter, $40 \mathrm{~mm}$ in length and a hemi-spherical cap. The body contains a small DC motor, a small polymer Li-Ion battery and a switch. The DC motor is a typical pager motor, which has a diameter of $6 \mathrm{~mm}$ and length of $10.4 \mathrm{~mm}$. The size of the Li-ion polymer battery is $17.3 \times 13.5 \times 3.7 \mathrm{~mm}^{3}$, and operates at $3.7 \mathrm{~V}$ with an energy storage capacity of $65 \mathrm{mAh}$, which is typically enough for a 4minute continuous operation of the robot. A six legged switch of dimensions $7 \times 3 \times 3 \mathrm{~mm}^{3}$ is used to connect the battery and the motor to control the power consumption. Figure 3 demonstrates the components placed in the head capsule of the robot.

The helical tail of the robot is made of steel wires with the diameter of $1 \mathrm{~mm}$ and apparent length of $48 \mathrm{~mm}$. The connection between motor and tail is made with a plastic sleeve coupling material, which is constructed by drilling from opposite sides with respect to diameters of the motor shaft and the helical tail. The plastic sleeve coupling is secured to the tail by applying a heat process to ensure a stable rotation of the tail.

Components in the glass capsule that makes of the body of the robot are assembled with a consideration of neutral buoyancy and symmetric weight distribution: a soft putty material is used for hand calibration as needed. The glass tube is sealed with a plastic cover to protect the components inside from direct contact with silicon oil. The plastic cap is drilled to fit the DC motor, and to provide a stable rotation of helical tail.

The swimming robot is placed inside a $30 \mathrm{~cm}-$ long tube with a diameter of $37 \mathrm{~mm}$ as seen from Figure 3. The tube is kept open-ended and placed inside a pool of silicone oil with dynamic viscosity of $5.6 \times 10^{-3} \mathrm{~Pa}$-s. Geometric properties of the body and twelve different helical flagella are listed in Tables I and II.

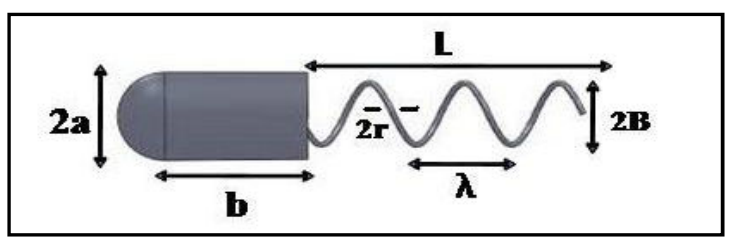

Fig. 1: Demonstration of prototype geometrical parameters

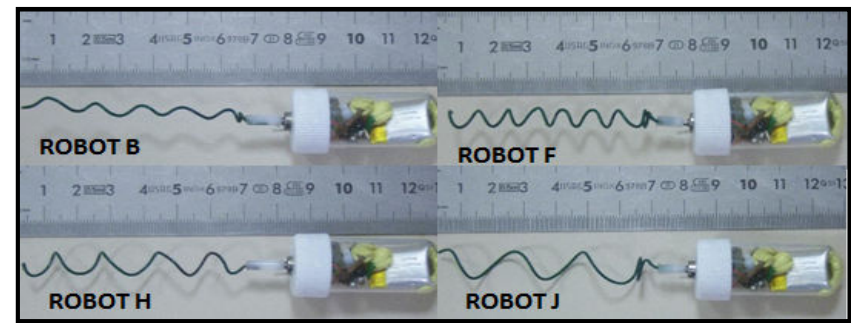

Fig. 2: Demonstration of prototypes Robot B, Robot F, Robot H, Robot J

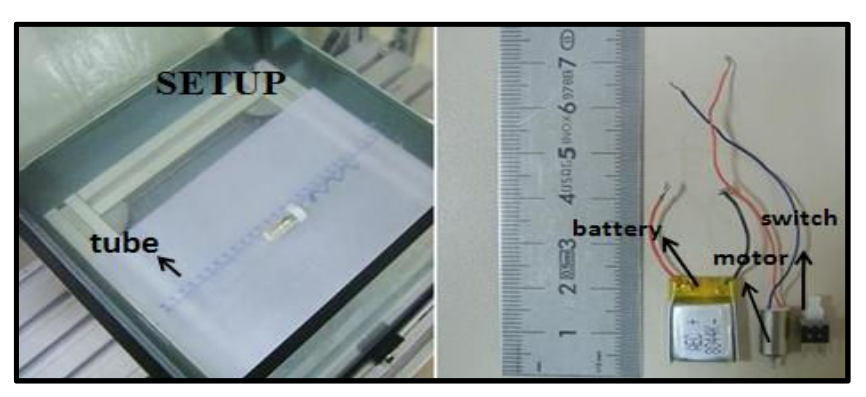

Fig. 3: Demonstration of Setup and Electronical Devices

TABLE I

COMMON DiMENSIONAL PROPERTIES FOR ROBOTS

\begin{tabular}{|l|l|}
\hline Radius of head, $\mathbf{2 a}$ & $15.8 \mathrm{~mm}$ \\
\hline Length of body, $\mathbf{b}$ & $32.1 \mathrm{~mm}$ \\
\hline Apparent length of tail, $\mathbf{L}$ & $48 \mathrm{~mm}$ \\
\hline Diameter of tail, 2r & $1 \mathrm{~mm}$ \\
\hline
\end{tabular}

Fig. 4 Demonstration of prototype geometrical parameters

TABLE II

DIMENSIONAL DATA FOR EACH ROBOT

\begin{tabular}{|l|l|l|l|l|l|l|l|l|l|l|l|l|}
\hline Robots & $\mathrm{A}$ & $\mathrm{B}$ & $\mathrm{C}$ & $\mathrm{D}$ & $\mathrm{E}$ & $\mathrm{F}$ & $\mathrm{G}$ & $\mathrm{H}$ & $\mathrm{I}$ & $\mathrm{J}$ & $\mathrm{K}$ & $\mathrm{L}$ \\
\hline $\begin{array}{l}\mathbf{2 B} \\
(\mathbf{m m})\end{array}$ & 2 & 2 & 2 & 4 & 4 & 4 & 6 & 6 & 6 & 8 & 8 & 8 \\
\hline $\begin{array}{l}\boldsymbol{\lambda}(\mathbf{m m}) \\
(\mathbf{m})\end{array}$ & 16 & 12 & 8 & 16 & 12 & 8 & 16 & 12 & 8 & 16 & 12 & 8 \\
\hline
\end{tabular}

For each case, experiments are repeated for at least three times, and average linear, $U$, and angular, $\Omega$, velocities, frequency, $f$, of the tail are calculated from the images in 2minute videos for each robot

\section{DESCRIPTION OF THE MODEL}

Mathematical model is obtained by assuming swimmer is submerged in an environment with no upstream velocity and without wall effects (contact forces) for robots that consist of two links connected with a simple revolute joint. We consider a 2 degree-of-freedom model for the linear velocity, $U$, of the robot and the angular rotation rate, $\Omega$, of the body. In the model, the angular velocity of the tail is assumed as given. The force balance can be given by: 


$$
\left[\begin{array}{l}
\boldsymbol{F}_{\text {body }} \\
\boldsymbol{T}_{\text {body }}
\end{array}\right]+\left[\begin{array}{l}
\boldsymbol{F}_{\text {tail }} \\
\boldsymbol{T}_{\text {tail }}
\end{array}\right]=\left[\begin{array}{l}
0 \\
0
\end{array}\right]
$$

Since the flow is in the viscous regime, we can assume that the force is proportional to the velocity with a simple relationship in the form $\mathbf{F}=\mathbf{D V}$ for the linear motion of the elliptical body.

$$
\left[\begin{array}{l}
\boldsymbol{F}_{\text {body }}^{s q r} \\
\boldsymbol{T}_{\text {body }}^{\text {sqr }}
\end{array}\right]=\left[\begin{array}{cc}
\boldsymbol{D}_{U}^{F} & 0 \\
0 & \boldsymbol{D}_{\Omega}^{T}
\end{array}\right]\left[\begin{array}{l}
\boldsymbol{U}_{\text {body }}^{\text {sqr }} \\
\boldsymbol{\omega}_{\text {body }}^{\text {sqr }}
\end{array}\right]
$$

Here $\mathbf{D}$ is a drag coefficient which represents the contributions from linear and angular velocities for an elliptical body [12]:

$$
\begin{gathered}
\boldsymbol{D}_{U}^{F}={ }^{4 \pi \mu b} /[\ln (2 b / a)-1 / 2] \\
\boldsymbol{D}_{\Omega}^{T}=\left(\frac{16}{3}\right) \pi \mu a^{2} b
\end{gathered}
$$

Here $\mu$ is the dynamic viscosity of fluidic environment, $a$ is minor radius and $b$ is major radius of elliptical body.

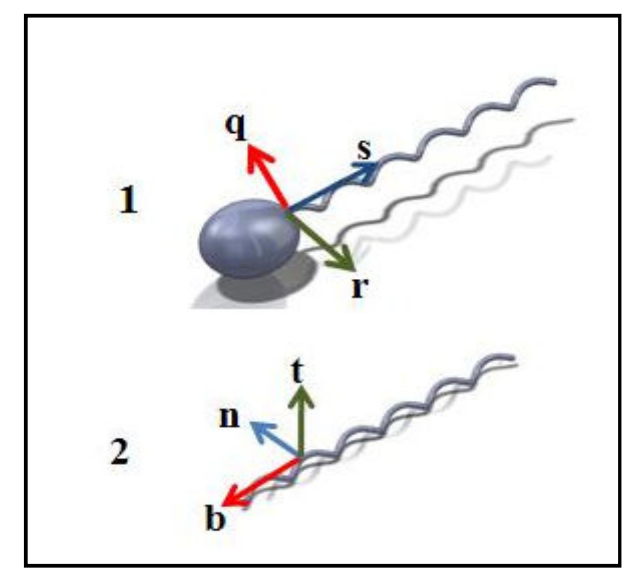

Fig. 5: Frames attached to parts of swimmer 1) Body coordinate frame of swimmer 2) Local Frenet Serret coordinate frame of helical tail

Rotational motion of the helix contributes to velocity of the body. This is modelled by RFT [14]. The position vector in body coordinates is given by $\mathbf{P}^{s q r}$ :

$$
\begin{gathered}
\boldsymbol{P}^{s q r}=\left[\begin{array}{c}
s \\
q(s, t) \\
r(s, t)
\end{array}\right]=\left[\begin{array}{c}
s \\
B_{q}(s) \sin (\omega t-k s) \\
B_{r}(s) \cos (\omega t-k s)
\end{array}\right] \\
B_{q}(s)=B_{q}^{\max }\left(1-e^{-c s}\right) \\
B_{r}(s)=B_{r}^{\max }\left(1-e^{-c s}\right)
\end{gathered}
$$

Here $\mathrm{k}=2 \pi \lambda$ which is wave number and $\omega=2 \pi f$ which is angular velocity of helical tail. In equation 2 and 3, $C$ is used as an envelope constant [14]. The velocity vector in body coordinates is $\mathbf{V}^{s q r}$ :

$$
\boldsymbol{V}^{s q r}=\frac{d p^{s q r}}{d t}=\left[\begin{array}{c}
0 \\
-B_{q}(s) \omega \sin (\omega t-k s) \\
B_{r}(s) \omega \cos (\omega t-k s)
\end{array}\right]
$$

The velocity in local Frenet-Serret coordinates on the helical tail is represented by $\mathbf{V}^{\text {bnt }}$ and relates to the local force on the tail $d \mathbf{F}^{b n t}$ as follow:

$$
d \boldsymbol{F}^{b n t}=\mathbf{C} \boldsymbol{V}^{b n t} d s
$$

Here $C$ matrix is the resistive coefficient matrix such as:

$$
\mathbf{C}=\left[\begin{array}{ccc}
-C_{n} & 0 & 0 \\
0 & -C_{n} & 0 \\
0 & 0 & -C_{t}
\end{array}\right]
$$

Local force matrix $d \mathbf{F}^{s q r}$ on body frame $s q r$ can be obtained with the rotation matrix from the Frenet-Serret coordinates to the body frame [14]. Total force on the body frame $\mathbf{F}^{s q r}$ is:

$$
\boldsymbol{F}^{s q r}=\int_{0}^{L} d \boldsymbol{F}^{s q r} d s
$$

Torque matrix of body is calculated similarly [14].

Because of the linearity of the Stokes flow, relationships between force, torque matrices and velocity matrices on tail can be obtained by [14]:

$$
\left[\begin{array}{c}
\boldsymbol{F}_{\text {tail }}^{\text {sqr }} \\
\boldsymbol{T}_{\text {tail }}^{\text {sqr }}
\end{array}\right]=\left[\begin{array}{cc}
\boldsymbol{C}_{U}^{F} & 0 \\
0 & \boldsymbol{C}_{\omega}^{T}
\end{array}\right]\left[\begin{array}{c}
\boldsymbol{U}_{\text {tail }}^{\text {sar }} \\
\boldsymbol{\omega}_{\text {tail }}^{\text {sqr }}
\end{array}\right]
$$

From equation (1) and (12) linear velocity and angular velocity can be calculated by specifying $\omega_{\text {tail }}$ which are calculated from experiments.

\section{RESULTS}

Robots' helical tails are twisted in counter clockwise direction. Thus, forward propulsion is ensured if the tail (body) rotates in clockwise (counter-clockwise) directions as seen from the front in Fig. 6. If the rotation directions are reversed, backward propulsion can be observed (see Fig. 6). Forward propulsion (in the direction from the tail towards the body) is due to the reaction force from the interaction between the rotating tail and the surrounding viscous fluid similar to the cork screw motion in solids. Note that this motion cannot generate propulsion in fluids where inertial forces are dominant (a.k.a high Reynolds number flows). The reaction force on the tail from the fluid due to tail's rotation also acts on the whole body via the revolute joint between the tail and the 
body. The propulsion force of the tail is balanced by the drag force of the whole swimmer including the body and tail.

Similarly the torque generated by the motor acts in opposite directions on the tail and the body. In the robot, motor's rotor is connected to the tail, and the stator to the body. Thus, motor's total angular velocity $2 \pi f+\Omega$ is divided between body and tail such as $\Omega$ and $2 \pi f$, respectively.

In experiments, linear velocity of the robot and rotation rates of the body and the tail are measured. The rotation of the tail is fed into the model as an input, and model's outputs, linear velocity of the robot and the angular velocity of the body are compared with measurements.

Due to the non-standard shape of the body, and near-the bottom-wall swimming conditions of the robot in experiments, theoretical drag coefficients given by (3) and (4) are modified. Linear and angular drag coefficients of the body are calculated from a CFD simulation, which is carried out by means of a commercial software package, COMSOL Multiphysics [17]. According to simulations these coefficients are:

$$
\boldsymbol{D}_{U}^{F}=6.79(\mathrm{~kg} / \mathrm{s}) \text { and } \boldsymbol{D}_{\Omega}^{T}=8 \times 10^{-4}\left(\mathrm{~kg}-\mathrm{m}^{2} / \mathrm{s}\right)
$$

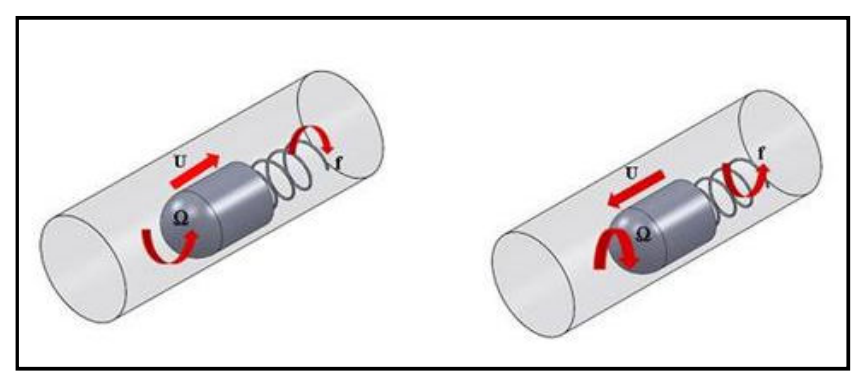

Fig. 6 Tail and body rotational directions for backward and forward propulsion

TABLE III

EXPERIMENTAL MEASUREMENTS

\begin{tabular}{|l|l|l|l|}
\hline ROBOTS & $\mathbf{U}(\mathbf{m} / \mathbf{s})$ & $\boldsymbol{\Omega} / \mathbf{2} \boldsymbol{\pi}(\mathbf{H z})$ & $\mathbf{f}(\mathbf{H z})$ \\
\hline A & $5.9 \mathrm{e}-4$ & 0.03527 & 4.322 \\
\hline B & $4.67 \mathrm{e}-4$ & 0.03443 & 4.325 \\
\hline C & $5.55 \mathrm{e}-4$ & 0.0428 & 5.133 \\
\hline D & $8.3 \mathrm{e}-4$ & 0.055 & 3.27133 \\
\hline E & $9.6 \mathrm{e}-4$ & 0.05667 & 3.144 \\
\hline F & $5.67 \mathrm{e}-4$ & 0.05697 & 2.843 \\
\hline G & $10.7 \mathrm{e}-4$ & 0.06457 & 2.49433 \\
\hline H & $9.44 \mathrm{e}-4$ & 0.05743 & 2.137 \\
\hline I & $4.44 \mathrm{e}-4$ & 0.04777 & 1.588 \\
\hline J & $9.7 \mathrm{e}-4$ & 0.0625 & 1.1833 \\
\hline K & $7 \mathrm{e}-4$ & 0.055 & 1.1 \\
\hline L & $2.8 \mathrm{e}-4$ & 0.06167 & 0.51667 \\
\hline
\end{tabular}

Average forward velocity of the swimmer and the angular velocity of the body are measured for 12 robots as shown in Table III. Body's rotation rates from experiments are compared with calculations from the model as seen in Figure 7 using the drag coefficients obtained from the CFD simulation (indicated as 'Base model'), and with tuned rotational drag coefficient (indicated as 'Tuned model'). Bars in the figure show one standard deviation of the measurements. Experi- mental measurements and calculations based on drag coefficients obtained from the CFD simulations have the same trend. Thus, we used a tuning coefficient for the rotational drag of the body to include additional friction between the body and the channel walls. This tuning factor is obtained as $1 / 3.03$ from the inspection of data, and used to modify the rotational drag as, $\mathbf{D}_{\Omega}^{T}=2.64 \times 10^{-4}\left(\mathrm{~kg}-\mathrm{m}^{2} / \mathrm{s}\right)$. Note that the tuning of the rotational drag coefficient of the body modified only the angular velocity of the body in calculations; linear velocity of the robot remained unaffected by the tuning of the body's rotational drag. According to Table IV, only robots A $(33.65 \%), \mathrm{C}(3.9 \%), \mathrm{G}(33.65 \%)$ and L (error 63.83\%) remain outside the 1-sigma envelope around the measurements: in fact, angular velocity of the robot $\mathrm{C}$ is also very close, but the discrepancy is very large for robots $\mathrm{G}$ and $\mathrm{L}$.

TABLE IV

StANDARD DEVIATIONS AND ERRORS In PERCENTAGE

\begin{tabular}{|c|c|c|c|c|}
\hline Robots & $\boldsymbol{U}_{\text {std }}( \pm \mathbf{\%})$ & $\boldsymbol{U}_{\text {error }}(\%)$ & $(\Omega / \mathbf{2 \pi})_{\text {std }}( \pm \%)$ & $(\Omega / \mathbf{2 \pi})_{\text {error }}(\%)$ \\
\hline A & 3.9 & 0.5 & 6 & 33.65 \\
\hline B & 7.13 & 77.13 & 28.4 & 25.35 \\
\hline C & 3.46 & 171.6 & 15.7 & 3.9 \\
\hline D & 8.43 & 68.5 & 8 & 9.81 \\
\hline E & 2.08 & 47.08 & 2.91 & 3.22 \\
\hline F & 5.87 & 87.67 & 23.5 & 10.65 \\
\hline G & 8.41 & 42.71 & 4.15 & 33.65 \\
\hline H & 1.43 & 20.26 & 23.5 & 23.1 \\
\hline I & 8.67 & 15.05 & 5.31 & 0.35 \\
\hline J & 3.09 & 17.74 & 6.72 & 1.28 \\
\hline K & 10 & 21.1 & 8 & 2.54 \\
\hline L & 7.14 & 54.6 & 2.67 & 63.83 \\
\hline
\end{tabular}

The average linear velocity of the robots are normalized by the wave prorogation speed on the tail's curvilinear coordinates, and compared with the normalized speed from the experiments in Figure 8. The wave propagation speed on the tail is given by [3]:

$$
V=f \lambda \alpha
$$

Here $f$ is the frequency of the rotation of the tail, $\lambda$ is the wavelength (helical pitch) and $\alpha$ is a coefficient that quantifies the ratio between the effective total length of the tail, $L_{e f f}$, and the helical length, such as [2]:

$$
\alpha=\frac{L}{L_{e f f}}
$$

Normalized average linear velocity for each robot agrees reasonably well: top three worst cases from Table IV are robots B $(77.13 \%), \mathrm{C}(171.6 \%)$ and $\mathrm{F}(87.67 \%)$. 


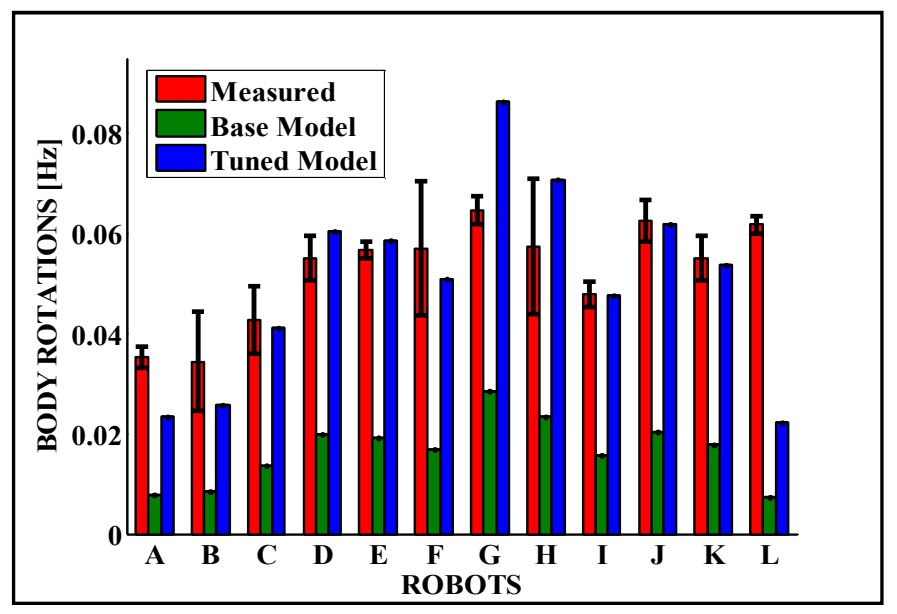

Fig. 7 Comparison of measured and calculated body rotations in $\mathrm{Hz}$

According to Lighthill's analysis, a free-swimming helical tail can reach only a percentage ( $\mathrm{ca} 30 \%$ ) of its wave propagation speed. In Figure 9, we plotted our measurements, and the results of the simple model with respect to a figure of merit that indicates the strength of the interaction of the tail with the viscous fluid, namely $B / \alpha=B L_{\text {eff }} / L$. As the radius of the helix, $B$, increases tail exerts more force on the fluid [16], and as $L_{\text {eff }} / L$ increases, the interaction between the tail and the fluid takes place on larger surface. Although, in the simple model, the normalized velocity of robots is limited by $10 \%$, experiments indicate that normalized velocity increases beyond that limit; robots reach up to $20 \%$ of its wave propagation speed.

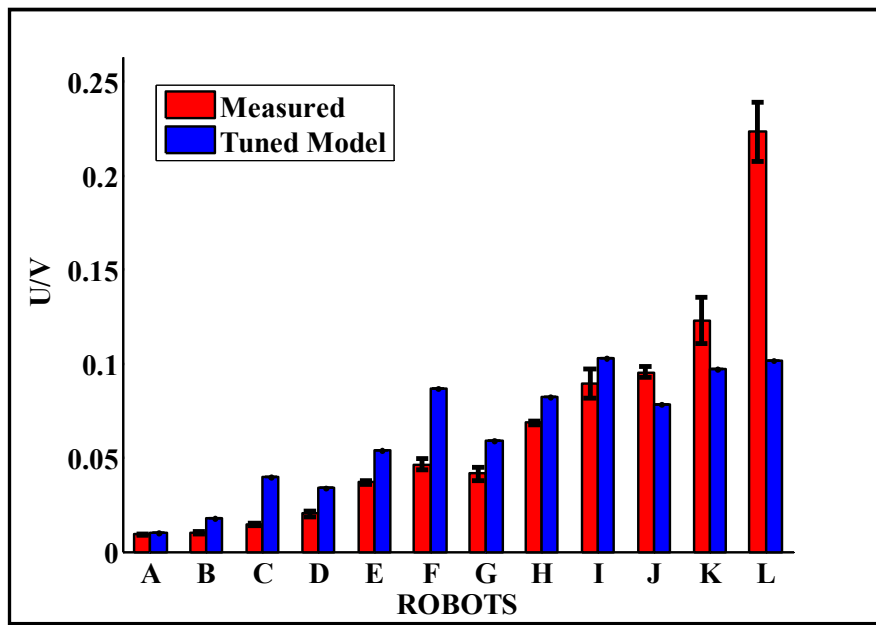

Fig. 8 Comparison of measured and calculated linear velocities nondimensionalized by the wave propagation speeds on helical flagella

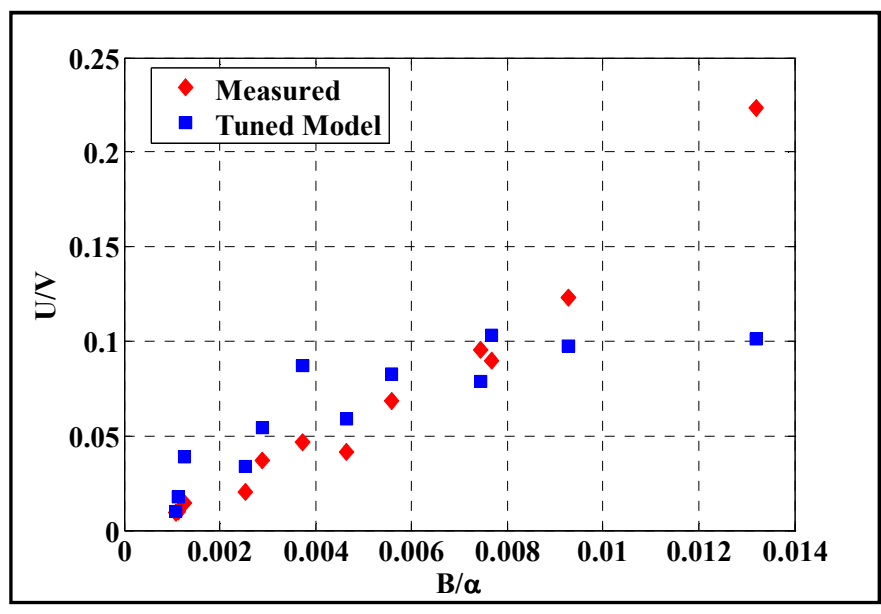

Fig. 9 Relation between $\mathrm{B} / \alpha$ and $\mathrm{U} / \mathrm{V}$

Shortcomings of the agreement between the results of the model and experiments could be due to experimental procedure, especially in balancing of the robot for all tails. Even if the balance of the body is achieved reasonably well in the silicone oil, tails, which are made of the metal wire, tend to introduce a bias in the motion of the robot. Thus the propulsion force from the tail is not always balanced by the drag along the axis of the channel, as the vertical component of the force balances the effect of gravity on the robot, and only the horizontal component propels the robot. For instance, in ROBOT L, the effective length becomes an important issue since the tail has the largest amplitude, and number of helical waves. As a result, it behaves differently in experiments with respect to others. Moreover, the resistive force coefficients from Lighthill's slender body theory do not take into account the presence of nearby channel walls. Thus, the coefficients that are obtained from the theory, which is developed for swimmers in infinite pools, may have applicability to the robots swimming in channels.

\section{V.CONCLUSION}

Experiments and modelling of onboard-powered autonomous swimming robots inside channels are presented in this paper. The swimming robot consists of a bullet shaped glass tube body and a helical tail which is made of steel wire. A revolute joint is used to transfer the motion between these two parts. Actuation (a DC motor), a switch and the power source of the robot is contained within the body.

Experiments are carried out in an open-ended tube which is filled by silicon oil. The measurements are done with respect to 12 different configurations of helical tail radii and helical pitch. The linear velocity of swimmer and angular velocities of the body and the tail are measured, and compared with the results of the theoretical mathematical model, which is based on the resistive force theory. Experimental results and calculations have the same trend for both the linear velocity of swimmer and angular velocity of body: only three of the robots remain outside the 1-sigma envelope around the measurements. Moreover, measured normalized average velocity for robots almost has the same trend with the calculated ones. 
Moreover, $B L_{e f f} / L$ is an important factor increasing normalized velocities which are calculated from experiments. On the other hand, for the calculated ones, linear velocity is stuck $10 \%$ of its wave velocity while $B L_{e f f} / \mathrm{L}$ is increasing. In general, results indicate that the motion of the swimmer can be predicted by means of the simple model reasonable well.

\section{REFERENCES}

[1] B. Nelson, I. Kaliakatsos, J. Abbott, "Microrobots for Minimally Invasive Medicine,"The Annual Review of Biomedical Engineering, pp. $55-85,2010$.

[2] Gray, J., Hancock, G.J., "The Propulsion of Sea Urchin Spermatozoa", J. Exp. Biol., Vol.32, pp. 802-814, 1955.

[3] Lighthill, J. 1976. Flagellar Hydrodynamics. SIAM Rev. 18:161-230.

[4] Keller, J.B., Rubinow, S. I., "Swimming of Flagellated Microorganisms", Biophysical Journal, Vol. 16, pp.151-170, 1976.

[5] M. Sendoh, N. Ajiro, K. Ishiyama, M. Inoue and K. I. Arai, "Effect of Machine Shape on Swimming Properties of the Spiral Type Magnetic Micro Machine," IEEE Transactions on Magnetics, vol 35, pp. 36883690, September 1999.

[6] S. Guo, Y. Hasegaw, T. Fukuda, and K. Asaka, "Fish Like Underwater Microrobot with Multi DOF," Proceedings of 200 International Symposium on Micromechatronics and Human Science, pp. 63-68, 2001.

[7] B. Kim, D. Kim, J. Jung and J. Park, "Undulatory Tadpole Robot Using Ionic Polymer Metal Composite (IPMC) Actuator," Proceedings of 2003 IEEE/RSJ International Conference on Intelligent Robots and Systems, pp. 2133-2138, 2003.

[8] R. Dreyfus, J. Baudry, M. L. Roper, M. Fermigier, H.A.Stone, and J. Bibette, "Microscopic artificial swimmwers," Nature, vol. 437, pp. 862-865, Oct. 2005.

[9] B. Behkam and M. Sitti, "E. Coli Inspired Propulsion for Swimming Microrobots,"Proceedings of the IMECE 2004, IMECE2004-59621, Nov.2004.

[10] B. Behkam and M. Sitti, "Bacterial flagella based propulsion and on/off motion control of microscale objects"

[11] L. Zhang, J. J. Abbott, L. Dong, K. E. Peyer, B. E. Kratochvil, H. Zhang, C. Bergeles, and B. J. Nelson, "Characterizing the swimming properties of artificial bacterial flagella," Nano Letters, vol. 9, no. 10, pp. 3663-3667, August 15, 2010.

[12] S. Chattopadhyay, X. Wu, "The Effect of Long Range Hydrodynamic Interaction on the Swimming of a Single Bacterium," Biophysical Journal, vol. 96, pp. 2023-2028, March 2009.

[13] J.B.Bates, G.R. Gruzalski, N.J. Dudney, C.F.Luck, X. $\mathrm{Yu}$,"Rechargeable Thin Film Lithium Batteries,"9th International Conference on Solid State Ionics, 1993.

[14] A. Tabak, S. Yesilyurt, "Validated Reduced Order Models for Simulating Trajectories of Bio-Inspired Artificial Micro Swimmer," Proceedings of ASME 2010 3rd Joint US-European Fluids Engineering Summer Meeting and $8^{\text {th }}$ International Conference on Microchannels and Minichannels, August, 2010.

[15] T. Goto, S. Masuda, K. Terada, Y. Takano, "Comparison between Observation and Boundary Element Analysis of Bacterium Swimming Motion," JSME International Journal, Vol.44, pp.958-963, 2001.

[16] A. Tabak, S. Yesilyurt, "Numerical Analysis of the 3D Flow Induced by Propagation of Plane Thin Membranes Inside Microchannels,"Proceedings on the 5th International Conference on Nanochannels, Mi crochannels \& Minichannels, 2007.

[17] COMSOL AB, Comsol Multiphysics User's Guide, 2010. 